\title{
Pedagogía de géneros textuales para fomentar engagement en la escritura académica en educación superior*
}

\author{
Genre-based Literacy Pedagogy to Develop Engagement in \\ Academic Writing in Higher Education
}

Teresa Benítez ${ }^{* *}$, Yanuacelly Guariguata ${ }^{* * *}$ y Adriana Pérez ${ }^{* * * *}$

\section{RESUMEN}

Este artículo presenta los resultados de un proyecto de investi-

Palabras clave: gación acción de corte cualitativo, implementado en una asignaaprendizaje activo, tura de formación básica en competencias comunicativas de la engagement, Universidad del Norte (Barranquilla-Colombia). El propósito fue analizar la contribución de la pedagogía de géneros textuales y las estrategias de aprendizaje activo en el engagement emocional y comportamental de los estudiantes; así como en su producción escritura académica, pedagogía de géneros textuales. escrita de un texto de explicación factorial. En el estudio participaron 63 estudiantes. Los datos fueron recolectados a través de observaciones de clases, entrevistas, análisis de documentos y encuestas. Los resultados demostraron incremento en el engagement emocional y comportamental, evidenciado en una participación activa e involucramiento en las actividades, cumplimiento de tareas y compromisos, interés e interacción activa con docentes y pares durante las actividades. Asimismo, los textos de los estudiantes al final del semestre confirmaron la apropiación

\footnotetext{
* Esta investigación está adscrita al proyecto: “La pedagogía de géneros en la enseñanza y aprendizaje de la competencia comunicativa académica en la Universidad del Norte", aprobado por la Dirección de Investigación, Desarrollo e Innovación (DIDI) de la Universidad del Norte.

** Colombiana. Magíster en Educación con énfasis en la enseñanza del inglés de la Universidad del Norte. Profesora asistente. Universidad del Norte, Barranquilla, Colombia. benitezt@uninorte.edu.co

*** Venezolana. Magíster en Literatura Latinoamericana de la Universidad Pedagógica Experimental Libertador. Profesora. Universidad del Norte, Barranquilla, Colombia. yguariguata@uninorte.edu.co

**** Venezolana. Doctora en Ciencias de la Educación de la Universidad Latinoamericana y del Caribe. Profesora asociada. Universidad del Norte, Barranquilla, Colombia. aderamirez@uninorte.edu.co
} 
de las características genéricas, léxico-gramaticales y semánticodiscursivas del género explicación factorial académico.

\begin{abstract}
This article reports the results of a mixed-method qualitative Action Research project conducted at Universidad del Norte (Barranquilla, Colombia) in an L1 Communicative Competence course. The main purpose of the project was to analyze the contribution of genre-based literacy pedagogy and active learning techniques in both students' behavioral and emotional engagement, as well as their performance in the writing classes. The sample included 63 students from three different groups. Data was collected through class observations, interviews, document analysis, and surveys. Results demonstrated increased behavioral and emotional engagement, evidenced in active participation and involvement in activities, fulfillment of duties, interest in activities, and interaction with teachers and peers. In addition, students' texts confirmed appropriation of the generic and linguistic characteristics of factorial explanation genre.
\end{abstract}

Keywords: Active learning, academic writing, engagement, genre-based literacy pedagogy. 


\section{Introducción}

La lectura y la escritura son competencias fundamentales para un óptimo desempeño en la universidad. Estas competencias facilitan la inserción de los estudiantes en las comunidades discursivas de las disciplinas y su participación exitosa en las actividades de producción y análisis de textos requeridas para aprender (Carlino, 2005). No obstante, el bajo nivel de competencias lectora y escrita con el que ingresan los estudiantes a la educación superior en Colombia ha sido motivo de preocupación y objeto de estudio en los últimos años (Colectivo Comunicaciones, 2002; González et al., 2015; Flórez y Cuervo, 2005; Pérez y Rincón, 2013), pues está asociado con el fracaso académico y la deserción universitaria (Benítez et al., 2018; Olave-Arias et al., 2013). Esta preocupación ha impulsado la implementación de estrategias para el fortalecimiento de dichas competencias en diversas universidades del país.

En la Universidad del Norte (Barranquilla-Colombia), se implementó el programa Eficacia Comunicativa (ECO), una estrategia institucional de alfabetización académica para desarrollar en los estudiantes las competencias comunicativas necesarias para aprender en la academia y enfrentarse con éxito a los retos que les impone el campo profesional. El programa, fundamentado en la teoría de género (Rose y Martin, 2012) y la pedagogía de géneros textuales de la Escuela de Sydney (Martin y Rose, 2005), incluye dos asignaturas de formación básica: Competencia Comunicativa I y II (CC1 y CC2) para los estudiantes del primer año de carrera. El proyecto de investigación que se reporta en el presente artículo se llevó a cabo en la asignatura CC1, que se imparte en el primer semestre a estudiantes de diferentes programas académicos. La asignatura tiene por objetivo desarrollar las competencias necesarias para comprender y producir textos propios de ambientes académicos. No obstante, alcanzar los objetivos del programa en 48 horas semestrales, específicamente los concernientes a la escritura académica, constituye un gran reto para algunos estudiantes debido a sus debilidades en esta competencia al iniciar su formación universitaria.

Datos recolectados por las docentes-investigadoras en 2018 revelaron un bajo desempeño en diferentes aspectos de la escritura, específicamente a nivel discursivo y léxico-gramatical, aspectos cruciales para lograr la claridad de los textos y alcanzar su propósito comunicativo. Los datos también evidenciaron estudiantes con una percepción ne- 
gativa hacia la escritura académica, sentimientos de frustración y rechazo por las tareas de escritura y poca afinidad con la metodología empleada, que son aspectos representativos del engagement emocional y comportamental.

Esta situación motivó el ajuste de las acciones pedagógicas en algunas fases del ciclo de enseñanza de escritura para generar engagement en los estudiantes y alcanzar los objetivos de escritura esperados. Por este motivo, se realizó el diseño e implementación de un proyecto de investigación acción (IA) con el propósito de analizar la contribución de la pedagogía de géneros textuales, potenciada con técnicas de aprendizaje activo, en el engagement y la producción escrita de los estudiantes. En este artículo se detallan las características del proyecto, la metodología de implementación y los resultados obtenidos.

\section{Marco teórico}

\section{Pedagogía de géneros textuales}

La pedagogía de géneros textuales es un enfoque metodológico para la enseñanza de la lectura y la escritura creado por investigadores de la Escuela de Sydney (Rose y Martin, 2012). Se enfoca en dos aspectos principales: el currículo y la pedagogía; por tanto, su objetivo principal es proporcionar a todos los estudiantes las herramientas necesarias para comprender y producir apropiadamente y de manera autónoma los géneros del currículo, a partir de la enseñanza explícita de su estructura y el lenguaje característico de los textos (Dreyfus et al., 2015).

Esta pedagogía se enmarca en la teoría de géneros de la lingüística sistémico-funcional (LSF) (Christie y Martin, 1997; Martin y Rose, 2008; Rose y Martin, 2012); la sociología de la educación (Bernstein, 1990; 1996) y el enfoque sociocultural del aprendizaje (Vygotsky, 1962). La teoría de géneros textuales es un enfoque funcional que concibe el lenguaje como un sistema para construir significados, y a los géneros textuales como fenómenos sociales con patrones reconocibles y propósitos definidos que pueden ser enseñados y aprendidos (Martin y Rose, 2008; Rose y Martin, 2012).

Por su parte, la teoría sociológica de Bernstein (1996) destaca el papel determinante de la escuela para potenciar o restringir el aprendizaje de los estudiantes. Concibe la enseñanza como un ciclo basado en 
una "pedagogía visible", en la cual se identifica claramente qué se debe aprender y qué se va a evaluar. En el enfoque sociocultural, el aprendizaje es concebido como un proceso social que sucede con el acompañamiento de personas expertas o en interacción con pares más aventajados (Vygotsky, 1962); dicho acompañamiento o andamiaje debe ser retirado gradualmente a medida que los estudiantes adquieren mayor confianza y logran un desempeño independiente (Bruner, 1987).

Esta pedagogía involucra un ciclo de enseñanza y aprendizaje (ver Figura 1), conformado por tres fases: deconstrucción de un texto modelo para asegurar la comprensión del contenido, su propósito social, su estructura y las características del lenguaje; construcción conjunta entre docente y estudiantes de un texto del mismo género, y construcción independiente de otro texto con las mismas características del modelo, cuando ya los estudiantes pueden hacerlo solos (Martin y Rose 2005; Martin, 1992; Rose y Martin, 2012).

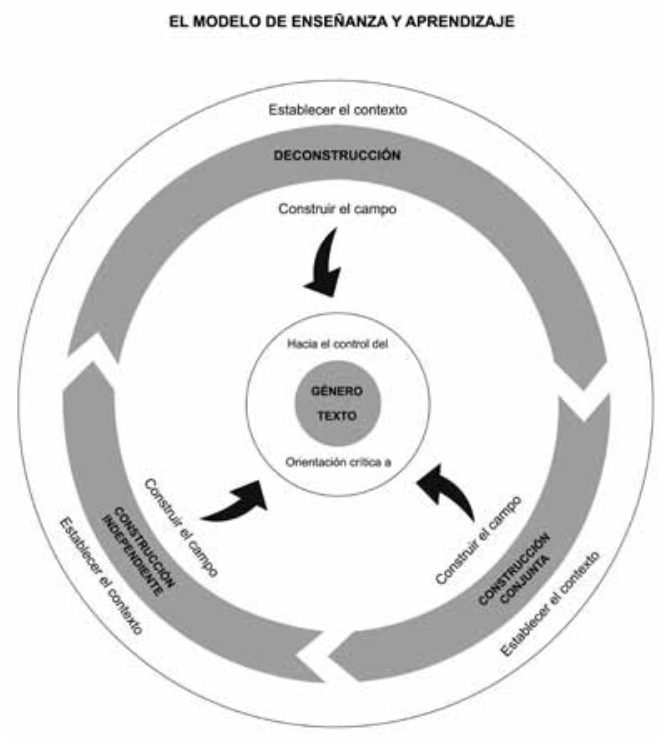

Figura 1. Ciclo de enseñanza y aprendizaje de la pedagogía de géneros textuales Fuente: Adaptado de Rothery y Steglin (1994, p. 8).

\section{Metodología leer para aprender}

El ciclo de enseñanza y aprendizaje de la pedagogía de géneros textuales ha sido adaptado de distintas maneras para responder a las nece- 
sidades específicas de los contextos en los que se aplica. Una de estas adaptaciones se manifiesta en la metodología leer para aprender (R2L por sus siglas en inglés) (Rose, 2004; Rose y Acevedo, 2006), que incorpora pasos adicionales de preparación para la lectura y la escritura en cada una de las fases del ciclo original para proporcionar un andamiaje más efectivo a los estudiantes antes del desempeño independiente (ver Figura 2).

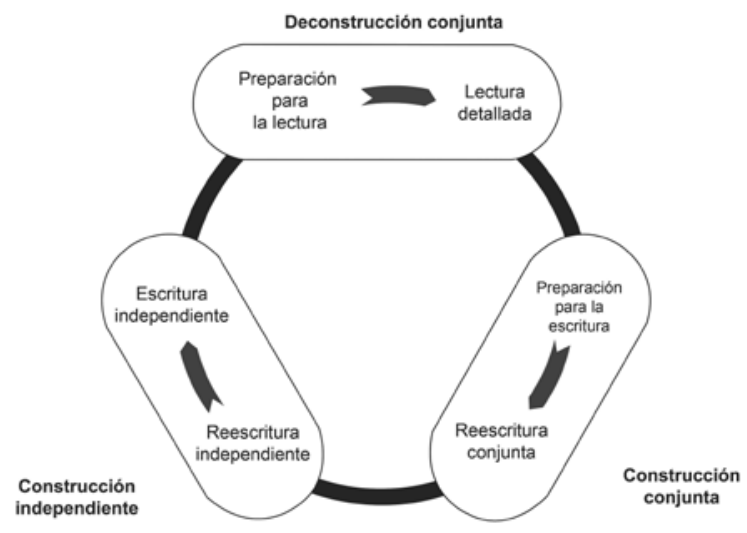

Figura 2. Fases del ciclo de enseñanza de la metodología leer para aprender (R2L)

Fuente: Adaptado de Rose (2004).

El andamiaje de la lectura y la escritura en cada una de las fases de la metodología R2L se lleva a cabo a través de un ciclo de interacción permanente en el aula, en el que el docente primero prepara a los estudiantes a través de preguntas para realizar la tarea exitosamente; una vez lo logran, evalúa las respuestas de los estudiantes y expande el conocimiento a partir de explicaciones, preguntas y diferentes tipos de elaboraciones. A este patrón de interacción (preparación, respuesta, evaluación y elaboración) se le denomina "ciclo de interacción para el andamiaje"1 (Rose y Acevedo, 2006), como se muestra en la Figura 3.

$1 \quad$ El término original en inglés asignado por Rose y Acevedo (2006) es: The scaffolding interaction cycle. 


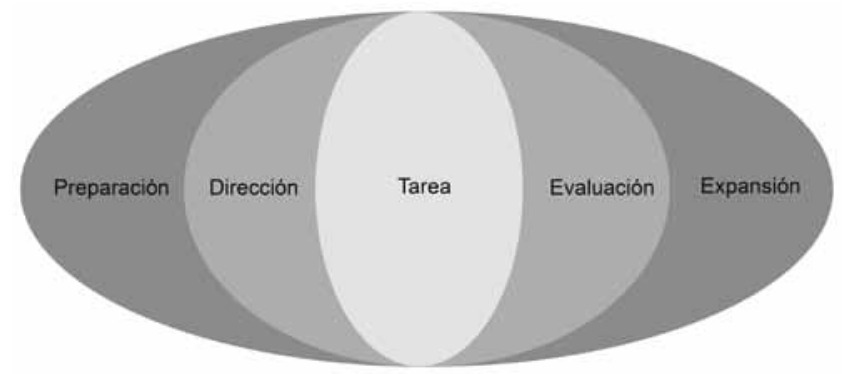

Figura 3. Ciclo de interacción para el andamiaje de la metodología leer para aprender

Fuente: Adaptado de Rose y Acevedo (2006).

Según los autores, en la primera etapa, preparación para la lectura, el docente realiza una contextualización sobre el tema, el género al que pertenece el texto, su propósito social y el área específica a la que pertenece. Asimismo, se hace una síntesis de cada una de las etapas o partes del texto, antes de la lectura. A este paso, en palabras de Acevedo, se le reconoce como "caminata por el texto". En la etapa de lectura detallada se realiza la lectura profunda del texto ${ }^{2}$, oración por oración, para llevarlos a la lectura independiente del mismo con un nivel de comprensión crítica. Esto se logra a través de diferentes tipos de preguntas que guían a los estudiantes hacia diferentes niveles de comprensión, desde la identificación de palabras y/o conceptos clave en cada oración (nivel literal), la deducción e inferencia de sus significados (nivel inferencial), hasta la interpretación crítica de dichos significados a partir de sus experiencias, conocimientos y valores (nivel crítico). Asimismo, en esta etapa se exploran las características del lenguaje del texto, orientando la reflexión de los estudiantes hacia la relación entre lenguaje y contexto y la manera en que el lenguaje contribuye al logro del propósito comunicativo.

La tercera etapa, preparación para la escritura, consiste en la toma de notas y organización de los conceptos clave identificados en el texto para la escritura de otro texto con las mismas características del modelo deconstruido. Las notas consideradas en esta etapa para la reescritura

2 La selección del fragmento en la lectura detallada dependerá de los objetivos que persiga el docente con el texto, ya sea para la comprensión del tema, o bien porque es un buen modelo para escribir el género (Rose y Acevedo, 2006). 
dependen del tipo de texto con el que se trabaje. Posteriormente, el docente guía la reescritura conjunta de otro texto con las mismas características del género analizado, a partir de la reelaboración de las notas escritas en el paso anterior. En la siguiente etapa de reescritura independiente, los estudiantes utilizan nuevamente las notas tomadas del texto original para producir sin ayuda un nuevo texto con las mismas características del modelo trabajado. Luego de realizado el andamiaje de la lectura y la escritura, los estudiantes son evaluados en la etapa de escritura independiente, en la que aplican los conocimientos adquiridos en la lectura y la escritura de nuevos textos, similares a los estudiados.

\section{Pedagogía de géneros textuales en contextos universitarios}

El ciclo de enseñanza y aprendizaje de la pedagogía de géneros textuales también ha sido adaptado para su aplicación en contextos universitarios. Dreyfus et al. (2015) incorporaron la fase de "construcción independiente negociada" al ciclo de enseñanza de escritura en un curso virtual de literacidad académica para estudiantes de Lingüística y Biología en una universidad de Hong Kong. En esta fase, el andamiaje se enfoca en la edición de los borradores de los textos escritos por los estudiantes, a través de diferentes niveles de retroalimentación.

Asimismo, en universidades argentinas se incorporaron las fases de “diseño y edición de los textos" al ciclo de enseñanza de escritura (Moyano, 2001; 2013; 2017), como se muestra en la figura 4.

\begin{tabular}{llll}
\hline \multirow{2}{*}{$\begin{array}{l}\text { Negociación de } \\
\text { campo }\end{array}$} & Deconstrucción & $\begin{array}{l}\text { - Lectura conjunta } \\
\text { - Lectura en pequeños } \\
\text { grupos } \\
\text { - Lectura independiente }\end{array}$ \\
\cline { 2 - 4 } & Diseño del & Construcción & $\begin{array}{l}\text { - Escritura conjunta } \\
\text { - Escritura en pequeños } \\
\text { grupos } \\
\text { - Escritura independiente }\end{array}$ \\
\hline \multirow{2}{*}{$\begin{array}{l}\text { Determinación } \\
\text { del contexto }\end{array}$} & Edición & $\begin{array}{l}\text { Edición conjunta } \\
\text { - Edición en pequeños } \\
\text { grupos } \\
\text { - Edición independiente }\end{array}$ \\
\hline
\end{tabular}

Figura 4 . Modelo pedagógico de enseñanza de escritura en universidades argentinas

Fuente: Adaptado de Moyano (2011). 
En la primera fase, los estudiantes aprenden a elaborar esquemas para organizar la información a incluir en los textos, antes de la escritura conjunta e independiente. En la segunda, docentes y estudiantes evalúan conjuntamente una muestra de los textos producidos por los estudiantes en la etapa de producción individual con el fin de editarlos. Este escrito es evaluado en términos de género, registro, realización lingüística, contenido, organización de la información, cohesión y aspectos formales, con ayuda de una rúbrica. El objetivo de esta etapa es proporcionar a los estudiantes herramientas metalingüísticas y metacognitivas para el análisis crítico de sus textos y para lograr un mayor control del género (Moyano, 2007).

Por su parte, el ciclo pedagógico para la enseñanza de la escritura implementado en la Universidad del Norte conserva las fases de "preparación para la lectura" y "preparación para la escritura" de la metodología R2L, e incorpora las fases de "diseño", "construcción" y "edición" del modelo argentino para la escritura de un texto del género explicación factorial (ver Figura 5). 


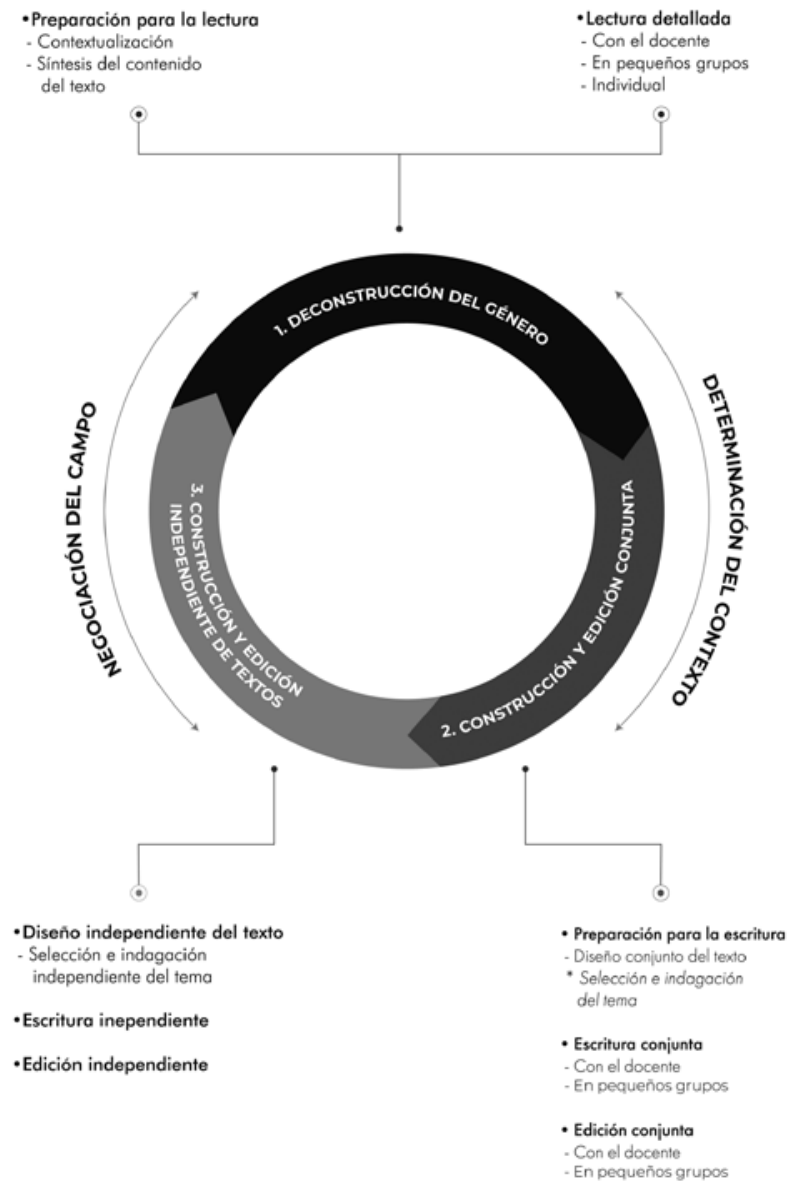

Figura 5. Modelo pedagógico de enseñanza de escritura en la Universidad del Norte

Fuente: Elaboración propia

Esta fusión favorece la apropiación de las características genéricas, léxico-gramaticales y semántico-discursivas del texto, así como la autonomía en la planeación, escritura y edición de los textos. En el apartado de metodología se presentará una descripción detallada del ciclo de enseñanza de escritura implementado en este proyecto.

\section{Explicación factorial}

Dada la naturaleza del curso en el que se implementó este proyecto, el análisis del funcionamiento del lenguaje en contexto constituye el cen- 
tro de instrucción. En este sentido, los géneros textuales son el insumo fundamental para construir conocimiento en la asignatura. Según Martin (1992), los géneros textuales son procesos sociales que obedecen a propósitos comunicativos y se construyen en pasos o etapas. Bajo esta concepción de género textual, se proporciona una clasificación de los géneros elementales que los estudiantes deben aprender a lo largo de su vida escolar, entre los que se cuentan géneros expositivos como los procedimientos, los informes y las explicaciones (Rose y Martin, 2012). Las acciones en este proyecto se enfocan en este último grupo de géneros.

En la universidad y en el campo profesional las explicaciones pueden aparecer incrustadas en macrogéneros, es decir, textos cuyo propósito social requiere la integración de diferentes géneros (Rose y Martin, 2012). El alto grado de abstracción que encierra el lenguaje que caracteriza las explicaciones, especialmente en textos académicos y profesionales, hace más difícil su comprensión y producción para los estudiantes que otros tipos de texto, por ejemplo, los narrativos (Barreyro et al., 2016). Por tanto, aprender a comprender y producir este género es esencial para los estudiantes universitarios, ya que este constituye una herramienta fundamental para el aprendizaje y la construcción de conocimiento en las disciplinas (Kendeou y van den Broek, 2005; Zwaan y Rapp, 2006).

Existen diferentes tipos de explicaciones: secuenciales, causales, de ciclos, consecuenciales y factoriales (Derewianka y Jones 2016; Martin y Rose, 2008). Este proyecto se enfoca en el género explicación factorial (FE), que explica un fenómeno en función de los factores que lo causan (Martin y Rose, 2008). Su estructura esquemática está conformada por dos etapas principales: identificación del fenómeno y explicación de los factores que lo causan. Se caracteriza por un lenguaje abstracto y fáctico, con predominio de participantes abstractos, procesos relacionales y materiales. Asimismo, predominan las relaciones lógicas de definición y causa-efecto, realizadas por frases verbales y preposicionales (Derewianka y Jones, 2016). La importancia del género explicación en educación superior y la complejidad que encierra para los estudiantes su comprensión y producción justificaron la implementación del ciclo propuesto para su enseñanza. 


\section{Aprendizaje activo}

El aprendizaje activo concibe a los estudiantes como individuos activos y conscientes de su proceso de aprendizaje. Según Brame (2016), el aprendizaje activo propicia la construcción de conocimiento a través de la ejecución de tareas que involucran tanto el pensamiento de orden superior como la metacognición. Esta metodología pone de manifiesto la importancia de la relación entre pares y la resolución de tareas complejas con ayuda de un par experto. Una de sus técnicas más efectivas es el aprendizaje colaborativo (Brame, 2016); así, el aprender se convierte en una dinámica multifocal y compartida para el individuo.

En una clase donde se aplique aprendizaje activo, el estudiante hace mucho más que observar, escuchar y tomar apuntes (González, 2000). Las clases integran la explicación, la demostración y el modelado por parte del docente con actividades de aprendizaje que involucran trabajo colaborativo, discusiones y resolución de tareas con diferentes grados de complejidad. Por este motivo, se considera una metodología apropiada para el contexto universitario. En el aprendizaje activo, la creatividad del docente y los recursos que utilice para lograr la interacción y participación activa de todos los estudiantes juegan un papel fundamental. Estos recursos incluyen lecturas, organizadores gráficos, recursos tecnológicos, entre otros (Sivan et al., 2010). Para este estudio, se incorporaron técnicas de aprendizaje activo al ciclo de enseñanza de escritura, con el fin de reforzar el andamiaje en la elaboración de textos académicos expositivos e incentivar el engagement de los estudiantes durante el proceso. Entre las técnicas incorporadas están: trabajo colaborativo, organizadores gráficos (de contenido, forma y función), y recursos tecnológicos para incentivar la interacción durante las actividades (foros de discusión virtuales, documentos interactivos y rúbricas de evaluación interactivas).

Las investigaciones en torno a la implementación de esta metodología en diferentes disciplinas universitarias confirman su efectividad para fomentar clases más inclusivas y productivas (Brame, 2016), así como el desarrollo de autonomía en el aprendizaje, pensamiento crítico y habilidades de lectura y escritura (Sivan et al., 2010). Sin embargo, la información acerca de sus aplicaciones para fomentar engagement 
en las clases de escritura en cursos de literacidad académica es escasa. Por tal motivo, se espera que este artículo pueda contribuir al estado del arte en el tema.

\section{Engagement}

El engagement (compromiso o involucramiento) de los estudiantes en el proceso de enseñanza y aprendizaje en educación superior se ha convertido en el foco de investigación de numerosos expertos en las últimas décadas. Resultados de investigaciones en este campo dan cuenta de la relación, ya sea directa o indirecta del engagement con el rendimiento académico (Carini et al., 2006; Gunuc, 2014), y demuestran su influencia en la participación activa de los estudiantes, el desarrollo de altos niveles de aprendizaje, el desarrollo personal y la retención en el sistema educativo (Umbach y Wawrzynski, 2005).

Las diferentes definiciones de este concepto involucran dos actores fundamentales: los estudiantes y la institución. En este sentido, el engagement se puede definir como la participación activa de los estudiantes en actividades (académicas o no académicas), ligadas al alcance de resultados de aprendizaje esperados por la institución y, asimismo, los esfuerzos de la institución por potenciar el compromiso de los estudiantes (Trowler y Trowler, 2010). Esto pone de manifiesto la estrecha relación entre los factores institucionales (académicos y no académicos) y el engagement. Es decir, implica participación, sentido de pertenencia y acciones por parte del estudiante, como respuesta a los procesos educativos que se llevan a cabo en las instituciones. Por lo tanto, entran en juego dimensiones que tienen tanto un polo positivo (el estudiante busca la potenciación de las actividades de aprendizaje) como un polo negativo (el estudiante busca entorpecer o interrumpir las actividades de aprendizaje) (Trowler y Trowler, 2010).

Algunos investigadores reconocen tres tipos de engagement: comportamental, emocional y cognitivo. En este proyecto solo se consideraron las variables: comportamental y el emocional.

Engagement comportamental. Se relaciona con la participación, esfuerzo, persistencia y búsqueda de ayuda encaminada a la comprensión y al aprendizaje (Fredericks et al., 2004; Linnenbrink y Pintrich, 2003b). Generalmente, está asociado a comportamientos observables 
como la participación en actividades académicas, sociales o extracurriculares; el cumplimiento de las normas de conducta; el manejo asertivo del comportamiento en clase; la participación activa en las discusiones y la realización de preguntas durante las clases. También se relaciona con la realización de tareas y el tiempo invertido en estas (Lawson y Lawson, 2013).

Engagement emocional. Se refiere a las reacciones afectivas, tanto positivas como negativas, a factores institucionales tales como los docentes, los pares, los contenidos curriculares y la misma institución. Entre estas reacciones se destacan interés, aburrimiento, disfrute, tristeza, ansiedad y sentido de pertenencia (Trowler y Trowler, 2010). En este tipo de engagement intervienen cuatro aspectos fundamentales que afectan el interés y el valor que dan los estudiantes a los procesos educativos: el interés personal; la percepción de utilidad (qué tan útil percibe el estudiante la actividad o contenido académico); las creencias sobre el valor o importancia del contenido /actividad/asignatura (qué tan importante es para su vida); y las experiencias emocionales positivas en su proceso de aprendizaje (Linnenbrink y Pintrich, 2003a).

\section{Metodología}

Este proyecto de IA es de corte mixto, porque integra técnicas cuantitativas y cualitativas para desarrollar el estudio (Gadner, 2012; Leech y Onwuegbuzie, 2009). Según Kemis y Mctaggart (1990), la IA es una forma de indagación autorreflexiva realizada por los participantes (docentes) de una situación social (educativa), con el fin de mejorar sus propias prácticas educativas, su comprensión de las mismas, y las situaciones e instituciones en que estas prácticas se realizan (aulas de clase o instituciones educativas). Se lleva a cabo en una espiral de ciclos de planificación, acción, observación y reflexión, en los que "el proceso de reflexión en la acción se convierte en un proceso de investigación en la acción" (Latorre, 2005, p. 5).

Existen diferentes modelos de implementación del ciclo de la IA (Elliot, 1993; Whitehead, 1991); en este proyecto se aplica el ciclo propuesto por Whitehead (1991), que comprende las siguientes etapas: identificar un problema en la práctica, planificar (diseñar la solución del problema), actuar (implementar la solución imaginada), observar 
(recoger datos de la implementación) y reflexionar (analizar datos y modificar a la luz de los resultados). A continuación, se describen las acciones realizadas en cada uno de los pasos del proyecto de IA implementado en el contexto objeto de estudio.

\section{Planificación: Diseño de la solución del problema}

Una vez identificado el problema en el contexto objeto de estudio, y luego de una indagación previa respecto de modelos y estrategias compatibles con nuestro enfoque metodológico, se diseñó una secuencia didáctica para la enseñanza de la escritura de un texto de explicación factorial académico. La secuencia diseñada fusiona fases de la metodología $R 2 L$ y del ciclo pedagógico para la enseñanza de la escritura descrito por Moyano (2013), con el fin de asegurar una mejor comprensión del contenido y las características lingüísticas del texto, así como autonomía en la planeación y edición de los textos. Asimismo, incorpora técnicas de aprendizaje activo en cada una de las fases del ciclo de andamiaje para incentivar la participación activa y el engagement de los estudiantes durante el proceso (ver Figura 6). Seguidamente, se diseñaron los materiales didácticos: guías de trabajo, organizadores gráficos y rúbricas interactivas, entre otros y los instrumentos para la recolección de los datos durante la implementación. 


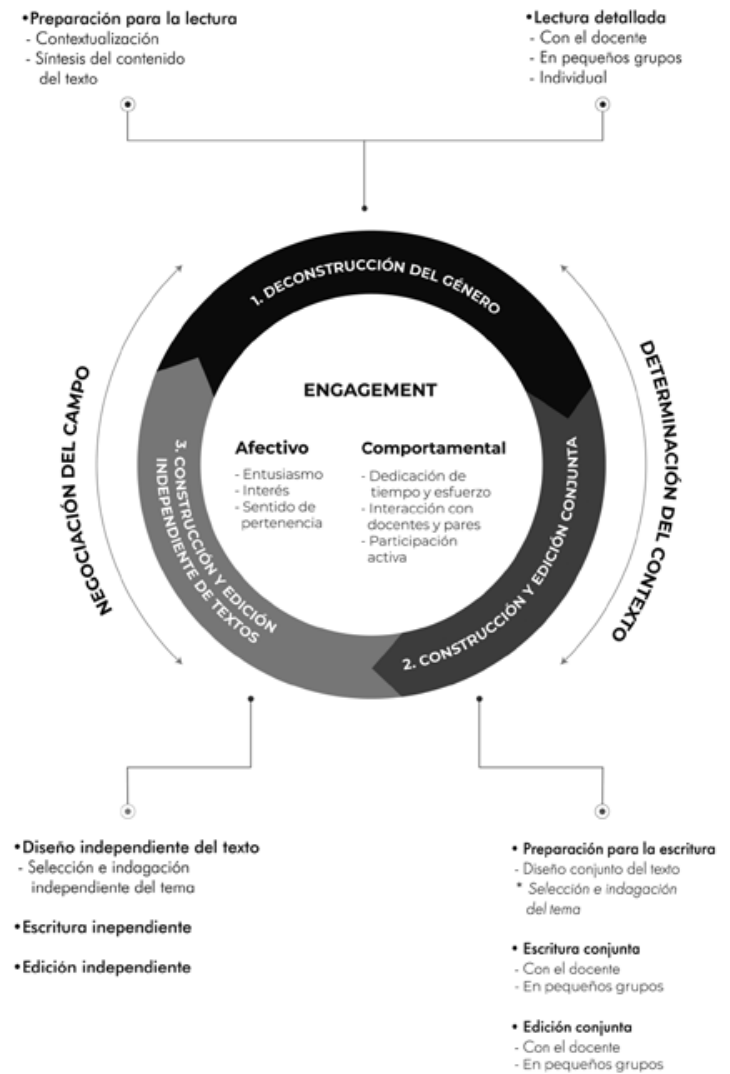

Figura 6. Ciclo pedagógico implementado en la Universidad del Norte Fuente: Elaboración propia.

\section{Acción: Implementación de la propuesta}

En esta etapa se implementó la secuencia didáctica en tres de los cursos impartidos por las docentes-investigadoras, y se recolectaron datos para su posterior análisis. En la implementación participaron 63 estudiantes de primer semestre, de diferentes programas académicos.

Para la deconstrucción del género se realizó, primero, la preparación para la lectura de un texto ejemplar y luego, la lectura detallada de las partes seleccionadas. En este paso, el andamiaje se enfocó en el reconocimiento de las características del género y una comprensión crítica del mismo, conforme con lo establecido en la metodología R2L (Rose y Acevedo, 2006). Seguidamente, en la etapa de construcción y 
edición conjunta, docente y estudiantes escribieron una nueva sección (fase) de explicación para agregar al texto modelo deconstruido. El andamiaje inició con el modelado del diseño del texto como preparación para la escritura, el cual consiste en la selección e indagación del tema del nuevo texto. Para ello, las docentes indican el tema del texto que se escribirá de manera conjunta y proporcionan a los estudiantes una tabla con información tomada de diferentes fuentes (a manera de notas) para elaborar el texto.

La escritura conjunta entre docente y estudiantes se desarrolló con la ayuda de un organizador gráfico compartido (ver Anexo 1), en el que se indicaron las fases del párrafo de explicación factorial a construir: identificación del grupo de factores a explicar (en un macro-Tema), identificación del factor específico a explicar en el párrafo (en un hiperTema), fases de explicación, conclusión y referencias.

El andamiaje se realizó a través de preguntas para guiar a los estudiantes a proporcionar la información necesaria para construir las diferentes fases del texto, haciendo énfasis en el manejo del lenguaje para su realización. El ejercicio se realizó in situ, en un documento compartido de Google Drive para que los estudiantes también aportaran sus ideas en el texto, luego de un proceso de negociación. Posteriormente, se llevó a cabo la edición conjunta del texto producido, paso en el que las docentes, también in situ, modelaron cómo evaluar y editar el texto según los parámetros de una rúbrica de evaluación.

A continuación, los estudiantes, en pequeños grupos, emularon el proceso anterior en un ejercicio de escritura conjunta de un nuevo texto del mismo género. Cada grupo recibió una guía de trabajo con la rúbrica de evaluación, un organizador de contenido con la información necesaria para incluir en el texto, y esquemas para organizar la información y estructurar sus textos según el género. Los textos fueron trabajados en un documento de Google Drive, compartido con los miembros del grupo y con las docentes, quienes guiaron la negociación de ideas y realizaron seguimiento a la contribución equitativa de cada integrante. Al finalizar, cada grupo autoevaluó su texto con la rúbrica de evaluación. Luego, estos textos fueron compartidos a través del foro de discusiones del catálogo virtual del curso en la plataforma Blackboard para recibir retroalimentación escrita de otro grupo, con base en los parámetros de la rúbrica. La evaluación y retroalimentación realiza- 
da por uno de los grupos fue socializada y discutida en clase, y usada como insumo para negociar alternativas para editar el texto.

Posteriormente, en la evaluación, los estudiantes, en parejas, realizaron la indagación de un nuevo tema para la escritura independiente de un texto de explicación factorial con las mismas características del texto analizado. En esta tarea aplicaron los conocimientos adquiridos en la selección, organización de la información, estructuración y manejo del lenguaje para lograr el propósito comunicativo del texto.

\section{Observación y reflexión}

Para la recolección de datos se observó un total de 13 clases, correspondientes a la implementación de la secuencia didáctica de escritura, las cuales fueron grabadas en video para su posterior análisis. Las observaciones también fueron registradas en notas de campo. Durante la implementación se realizaron entrevistas a 20 estudiantes después de las clases y se analizaron los correos electrónicos enviados por los estudiantes a las docentes, las retroalimentaciones escritas en los foros de discusión y sus producciones escritas. El corpus estuvo conformado por 15 textos escritos seleccionados aleatoriamente para ejemplificar las categorías en los resultados. Se utilizó una rúbrica de evaluación para evaluar los aspectos correspondientes a la escritura, según los objetivos del curso.

Al final de la implementación se aplicaron dos encuestas a los estudiantes, una para indagar su percepción acerca de la escritura académica y la metodología empleada $\left(\mathrm{QCD}^{3}\right)$, y la otra para determinar el engagement emocional y comportamental alcanzado con la implementación (encuesta NSSE ${ }^{4}$ adaptada). Los datos de las encuestas fueron registrados en una matriz de Excel para facilitar el análisis.

Una vez recolectada la información, esta fue organizada, categorizada y analizada a la luz de los objetivos del proyecto. Se triangulan

3 Diagnóstico rápido de curso (Quick Course Diagnosis, por sus siglas en inglés), recoge la percepción de los estudiantes acerca de una clase. El engagement evaluado en la encuesta apunta a la inclusión, el desafío óptimo y el apoyo a la autonomía.

4 Encuesta Nacional de Participación Estudiantil (NSSE, por sus siglas en inglés), revisa el engagement emocional, comportamental y cognitivo de los estudiantes en las actividades llevadas a cabo en la institución. En este proyecto, solo se tomó en cuenta el engagement emocional y comportamental. 
(Denzin, 1978) simultáneamente en este diseño los datos cualitativos y cuantitativos para responder la pregunta de investigación.

\section{Resultados}

\section{Compromiso de los estudiantes (engagement)}

Las actitudes y el comportamiento de los estudiantes en las diferentes actividades durante la implementación confirmaron la existencia de engagement (comportamental y emocional). Según Salonava et al. (2005) está caracterizado por el vigor, la dedicación y la absorción, así como la participación, el esfuerzo y la persistencia durante la realización de las tareas académicas. Evidencias de estas actitudes y comportamientos se encuentran en las observaciones de clases durante las interacciones con las docentes y entre pares en los diferentes trabajos grupales.

Tabla 1.

Ejemplo 1. Interacción docente-estudiante (contexto 3)

P ¿Quién quiere leer lo que escribió?

[un estudiante levanta la mano para participar] Vamos a ver. Inténtalo.

[otra estudiante levanta la mano]

Déjalo que lo intente. Léelo en voz alta, por favor [Hay ruido en la clase, la profesora llama la atención]...

Un segundo, vamos a prestarle atención al compañero, porque, entonces ¿cómo aprendemos? léelo en voz alta. Desde esta competencia "por otra parte"

E [Lee en voz alta] Por otra parte, Salonava, Safre, Graw, Martínez (2005) dentro del marco de la teoría social cognitiva hacen referencia a la competencia cognitiva como (...)una de las características trascendentales del desempeño académico.

$\mathrm{P} \quad$ Ok, o sea que acabas de subir la definición para acá [señala la parte del texto en el tablero]. Pregunto, ¿si hacemos ese ejercicio así, "el por otra parte" cabe? ¿Se puede usar?

EE No

P ¿Cierto que no? ¡Muy bien! Sí, es cierto, necesitas un conector, pero no puede ser por otra parte, porque por otra parte indica contraste y lo que necesitas es un conector que te indique similitud porque si ves, las dos teorías o los dos grupos de teóricos te están hablando prácticamente de lo mismo [señala la parte del texto en el tablero]. Entonces, ¿qué conector, Angie, podría ser? si no es por otra parte ¿qué conector sería?

\begin{tabular}{ll}
\hline $\mathrm{E}$ & Uno de adición \\
\hline $\mathrm{P}$ & ¡Uno de adición! Estoy completamente de acuerdo, ¿cuál?, \\
\hline $\mathrm{E}$ & Además \\
\hline $\mathrm{E}$ & O sea que de pronto eso estaba más enredado y así queda mejor \\
\hline
\end{tabular}

Fuente: Elaboración propia. 
La Figura 7 muestra la participación activa de los estudiantes durante la negociación de información en la construcción conjunta de la fase de explicación del texto. El ejercicio se realiza en un documento compartido por estudiantes y docentes en Google Drive. Se evidencia el engagement comportamental de los estudiantes en el manejo asertivo del comportamiento durante el ejercicio, su participación espontánea para responder o realizar preguntas y proponer alternativas, así como en el esfuerzo y la persistencia encaminada a la comprensión y al aprendizaje (Fredericks et al., 2004). En este caso las preguntas de la docente son el motor para promover la participación de los estudiantes a partir de la discusión, el análisis, y la reflexión.

Otro indicio de engagement comportamental se ilustra con la Figura 8, que muestra el mensaje de uno de los correos electrónicos enviados por un estudiante a una de las docentes.

\begin{abstract}
Muy buenas tardes profesora.
En la siguiente quiero ofrecerle disculpa por mi bajo rendimiento en lo que se lleva del curso, se han presentado un gran número de incovenientes tanto academicos como personales, lo cual no es excusa pero igual me disculpo. Además del punto mencionado anteriormente me gustaria presentarle una solicitud para poder hacer su próximo parcial más tarde, esto se debe a que hace ya varios días no me he sentido bien de salud y me estaré haciendo algunos examenes médicos a lo largo de la próxima semana, presento esta solicitud ya que quisiera tener la oportunidad de poder estudiar de la mejor manera y no creo que pueda hacerlo viendo como me siento, prometo dar mi mejor esfuerzo para el parcial y para lo que queda del semestre, me gustaría también organizar algún tipo de reunión en pro de poder ver en que puntos puedo mejorar en lo que respecta a su materia, obviamente en lo que esté a su alcance. Estaré pendiente a su respuesta y agradezco de antemano por su tiempo.
\end{abstract}

Figura 7. Ejemplo 2. Correo electrónico de estudiante\#11 (Contexto 2)

En este ejemplo se evidencia el sentido de responsabilidad del estudiante en la asignatura y su interés por el cumplimiento de los compromisos, además del tiempo invertido en su realización (Lawson y Lawson, 2013). También, es notoria la búsqueda de ayuda encaminada a la comprensión y el aprendizaje (Linnenbrink y Pintrich, 2003a) en la solicitud del estudiante a la profesora de concederle una reunión para aclarar dudas y plantear oportunidades de mejora. Asimismo, este ejemplo muestra evidencias de engagement emocional (Linnenbrink y Pintrich, 2003b) en la conexión positiva que demuestra el estudiante 
con la asignatura (y tal vez con la docente), al tomarse el trabajo de escribir un correo para ofrecer disculpas por el bajo rendimiento en la asignatura y comprometerse a un mejor desempeño.

Otros indicios de engagement emocional se encuentran en los documentos analizados. Esto se aprecia en las notas de campo que se registraron en las observaciones durante la construcción conjunta de un párrafo de explicación factorial en pequeños grupos.

La profesora pide a los estudiantes escribir una propuesta para evitar la repetición de palabras usando cualquiera de las estrategias de cohesión trabajadas en clase. Los estudiantes se muestran interesados en la actividad y participan rápidamente (...) La mayoría de los estudiantes tienen los celulares sobre el escritorio, pero no lo utilizan. Permanecen concentrados en la actividad.

La profesora toma los casos que le presentan y realiza retroalimentación a todo el grupo. Varios estudiantes intervienen en la retroalimentación para dar aportes (...).

Figura 8. Ejemplo 3. Notas de campo Observación \#2 (Contexto 1)

En el ejemplo se evidencia el interés de los estudiantes por la actividad en la concentración y la participación oportuna durante la realización de la tarea. Además, el hecho de desplazar el uso de sus teléfonos móviles a un segundo plano para dar prioridad a la elaboración de la tarea es un indicador de disfrute (Trowler y Trowler, 2010).

Otros indicios de reacciones afectivas a factores institucionales se encuentran en las entrevistas. Fragmentos de las entrevistas muestran las percepciones positivas de los estudiantes respecto de la utilidad y pertinencia de la escritura académica y la metodología implementada (ver Figura 10).

Considero que lo aprendido en esta materia es pertinente en mi carrera, ya que el saber escribir un texto académico de la mejor manera es vital para cualquier área de trabajo. Aparte, considero que, si en un futuro tuviera la necesidad de hacer un texto de similares características, sería capaz de realizarlo.

Figura 9. Ejemplo 4. Entrevista contexto 1

Igualmente, las entrevistas desvelan otras evidencias de engagement emocional como las actitudes de los estudiantes hacia el curso, los contenidos y las docentes (ver Figura 11). 
Me gustan estas clases, sé que son importantes para todas las escrituras en la universidad, la profesora nos aporta muchísimo y nos ayuda a ordenar las ideas que son necesarias para que me podamos redactar un texto expositivo. Ella siempre nos acompaña y siempre comenta cómo lo hicimos.

Figura 10. Ejemplo 5. Entrevista, contexto 2

Datos de las encuestas permiten corroborar las manifestaciones de engagement emocional y comportamental mencionadas anteriormente. En la Figura 12, las respuestas de los estudiantes en la encuesta NSSE muestran alto grado de engagement comportamental y emocional en la mayoría de los estudiantes.

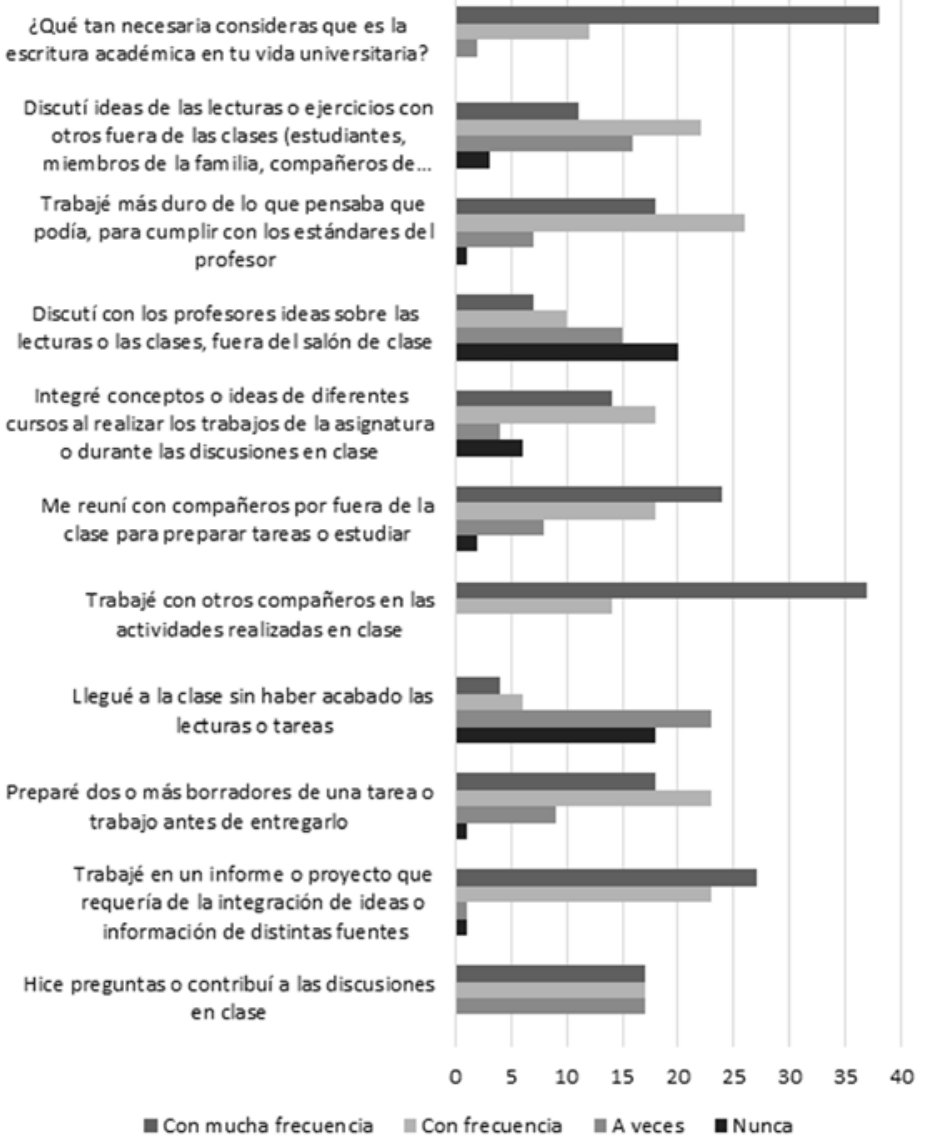

Figura 11. Encuesta NSSE

Fuente: Elaboración propia. 
Como se observa, las respuestas a las preguntas $1,2,3,7$, y 9, que indagan por el engagement comportamental, se ubican en los rangos "con mucha frecuencia" y "con frecuencia". Esto denota un alto grado de participación y compromiso de los estudiantes en las actividades académicas y se evidencia en la disposición de escucha y participación activa por parte de los estudiantes, que favorecen el desarrollo de la clase; en el acoplamiento a las estrategias pedagógicas y actividades desarrolladas en la clase; el seguimiento de instrucciones y aceptación de las recomendaciones brindadas por las docentes; y en el cumplimiento de tareas y compromisos dentro y fuera del aula para alcanzar los estándares requeridos.

Por otra parte, indicios de engagement emocional positivo se encuentran en las respuestas a las preguntas 5,6 y 10, que también se ubican en el rango de "con mucha frecuencia" y "con frecuencia”. Estas demuestran el sentido de identificación y conexión de los estudiantes con sus pares, interés por el correcto ejercicio de las actividades académicas, y, en cierto modo, disfrute por su realización.

Asimismo, en las respuestas proporcionadas por los estudiantes en la encuesta QCD (ver Figura 13), se observa la percepción de los estudiantes respecto de la inclusión, el desafío óptimo y el apoyo a la autonomía durante las clases, teniendo en cuenta los descriptores de Reeve (2003).

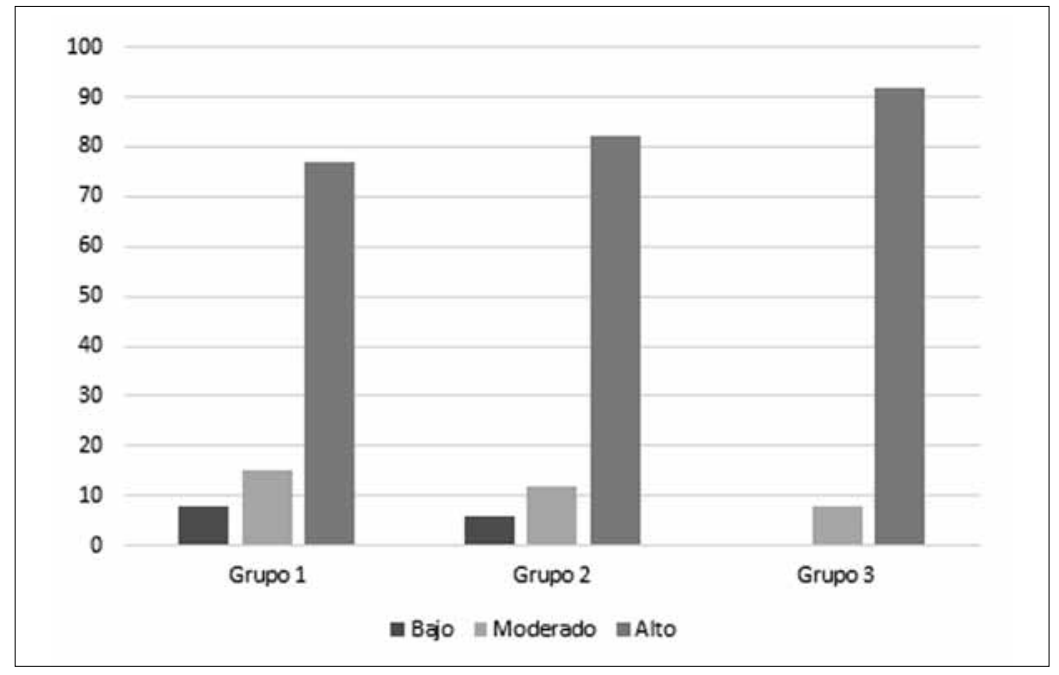

Figura 12. Resultados engagement encuesta QCD grupos 1, 2 y 3 Fuente: Elaboración propia. 
Tal como lo manifiesta Reeve (2003), la inclusión es una necesidad básica del ser humano que influye de forma directa en la motivación y el engagement del estudiante, en tanto le permite conectarse emocionalmente e involucrarse interpersonalmente en vínculos cálidos, interesados y sensibles, no solo con sus compañeros de clase sino también con el docente. Por su parte, la percepción de desafío en las tareas favorece la necesidad básica de competencia, relacionada con el deseo de ser eficaz en alguna actividad; de esta manera, si el aprendiz ubica la tarea como muy fácil de realizar o muy difícil de comprender, el esfuerzo invertido y, por tanto, el compromiso con la misma se verá disminuido. Por último, el apoyo percibido de la autonomía durante la clase es favorecido por experiencias de aprendizaje que impliquen control interno de las causas que controlan las situaciones, sentido de sensibilidad psicológica y control de sus acciones.

\section{Competencias de escritura académica desarrolladas por los estudiantes}

Los resultados del análisis de las características de los textos escritos por los estudiantes durante la escritura independiente permitieron confirmar la contribución de la propuesta implementada en sus competencias de escritura. En el análisis se tuvieron en cuenta las siguientes categorías: configuración de la estructura esquemática del texto (etapas y fases); selección del lenguaje para construir las etapas y fases del género explicación factorial; evolución del registro formal; cohesión y coherencia.

En cuanto a la estructura, los resultados demuestran que la mayoría de los estudiantes logró estructurar sus textos según los parámetros establecidos en la clase para el género explicación factorial académico: título, identificación del fenómeno en un macro-Tema, explicación de factores y conclusión de la explicación en un macroNuevo (ver Anexo 2).

Asimismo, las calificaciones obtenidas en cada aspecto correspondiente al género (estructura esquemática y propósito comunicativo) de la rúbrica de evaluación (ver Anexo 3) corroboran que la mayoría fue capaz de realizar las etapas y fases del género y configurar el propósito comunicativo en cada una de ellas, como se muestra en la Figura 14. 


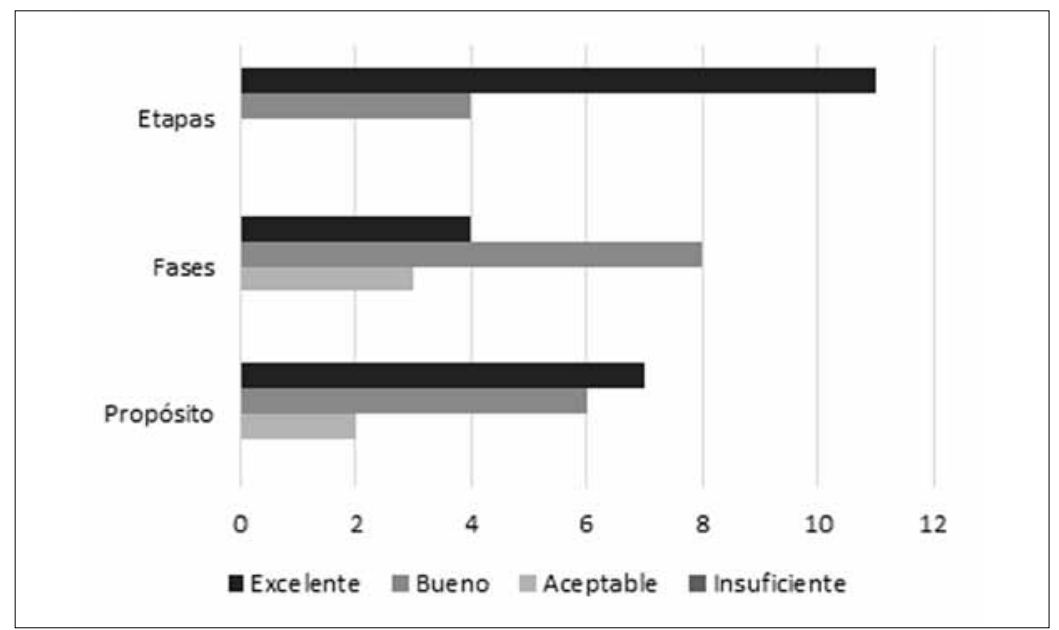

Figura 13. Resultados realización de etapas y fases en el texto Fuente: Elaboración propia.

Por otra parte, las expresiones marcadas en negrita en Figura 15 evidencian la apropiación de expresiones para construir las definiciones, conceptualizaciones y relaciones de causa-efecto, propias del género explicación factorial (Derewianka y Jones, 2016).

El estrés se puede definir como "el resultante de la presión que una fuerza externa ejerce sobre un cuerpo, creando una reacción interior (...)" (Sandin, 1995). En el caso del estrés académico esto ocurre como respuesta a la presión que (...). Existen diferentes factores asociados al estrés académico, entre los cuales resaltan los (...).

Figura 14. Ejemplo 6. Fragmento del texto \# 5. Contexto 1

Asimismo, en la Figura 16 se observa una evolución del registro informal con el que ingresan los estudiantes de primer semestre, a un registro formal, congruente, propio de los textos explicativos. Este se caracteriza por el alto grado de abstracción manifestado en la precisión léxica para adecuar el registro al campo del texto.

El asma es una enfermedad crónica que produce la inflamación de las vías respiratorias, debido a la gran infiltración celular de la pared bronquial. Debido a la inflamación de esta se producen episodios recurrentes, en niños $\mathrm{y}$ adultos, de problemas respiratorios como: ahogamiento, sibilancia, tos y dolor torácico.

Figura 15. Ejemplo 7. Fragmento del texto \# 17 (Contexto 3) 
En el ejemplo se marcaron en negrita las evidencias de precisión léxica. Son términos acordes al contenido del texto y propios del campo disciplinar al que pertenece, lo que demuestra indagación del tema para construir adecuadamente el campo en el texto. Esto se debe a que aprenden a utilizar el lenguaje "no solo para la obtención de un beneficio educativo, sino para el logro de una mayor confianza en sí mismos y una mejor conexión con el entorno" (Álvarez y Parra, 2015, p. 9).

Por su parte, en la Figura 17 se observa el empleo de nominalizaciones $^{5}$ (resaltadas en negrita) que contribuyen a dar objetividad y condensar información en estructuras complejas (Martin y Rose, 2008), las cuales eran expresadas en varias oraciones simples en los primeros intentos de escritura.

Por otra parte, expertos sostienen la existencia de factores ligados a causas antrópicas, que causan terremotos. Ejemplos de esto serían los terremotos originados por la minería ilegal y la extracción de petróleo, que pueden lograr ocasionar micro-terremotos o terremotos. En primer lugar, a esta práctica de extracción se le denomina "Fracking". El fracking, o fracturación hidráulica, es un (...).

Figura 16. Ejemplo 8. Fragmento del texto \#9. Contexto 2

Los resultados también muestran evolución en la habilidad para organizar la información a lo largo del texto y en el uso de mecanismos para lograr la coherencia. En la Figura 18 (análisis adaptado de Dreyfus et al., 2015), la coherencia se manifiesta en la manera en que se organiza la información a lo largo del texto a través de macro-Temas ${ }^{6}$ e hiper-Temas ${ }^{7} \mathrm{y}$ así proporcionar pistas para anticipar la idea global. Esto se reconoce como periodicidad, que se refiere al flujo periódico de la información para crear un texto coherente y con sentido (Rose y Martin, 2012).

5 Nominalización: procedimiento léxico-gramatical que consiste en sustantivar verbos y adjetivos.

6 Macro-Tema: Parte al inicio de un texto que identifica el tema que se va a desarrollar en el texto.

7 Hiper-Tema: Oración al inicio de un párrafo o sección en el texto que anticipa el tema que se desarrollará en la sección. Estos términos son traducidos a partir de Halliday (1994). 


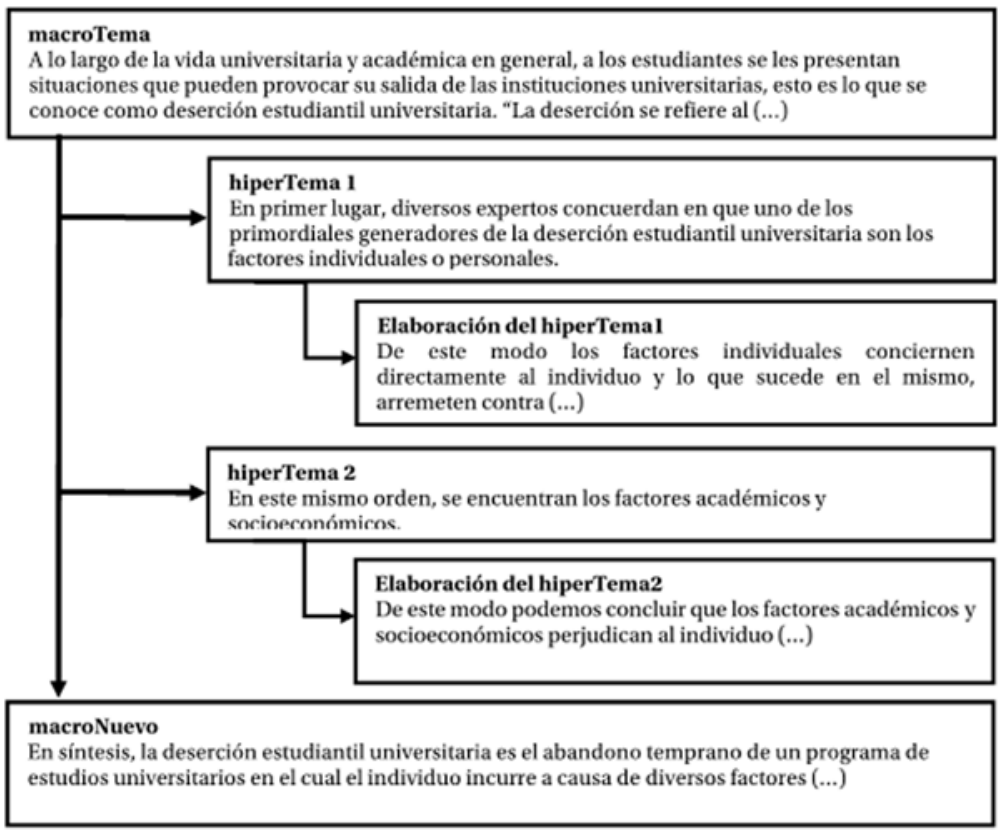

Figura 17. Periodicidad en el texto\#8 (Contexto 2)

Fuente: Adaptado de Dreyfu et al. (2015).

El uso de recursos para lograr coherencia se evidencia en los patrones de progresión temática, los cuales contribuyen a que la información avance lógicamente y se mantenga un hilo conductor en el despliegue de la información. En la Figura 19 se presenta un fragmento del macro-Tema que explica los factores que causan la deserción universitaria. Esta etapa del texto está conformada por fases de identificación del fenómeno (oración 1) y contextualización (oraciones 2 y 3$)$.

(1) A lo largo de la vida universitaria y académica en general, a los estudiantes se les presentan situaciones que pueden provocar (...). (2) "La deserción se refiere al abandono prematuro de un programa de estudios antes de alcanzar el título o (...)" (Himmel, 2002). (3) La deserción estudiantil universitaria es común en los jóvenes y se produce principalmente porque los estudiantes (...).

Figura 18. Ejemplo 9. Fragmento del texto \#8. Contexto 2

Para determinar cómo despliega la información en las diferentes fases y en qué información enfatiza en las diferentes oraciones obser- 
vamos los patrones de progresión temática empleado por los estudiantes en el texto a partir del análisis de Tema y Rema $^{8}$ (ver Figura 20).

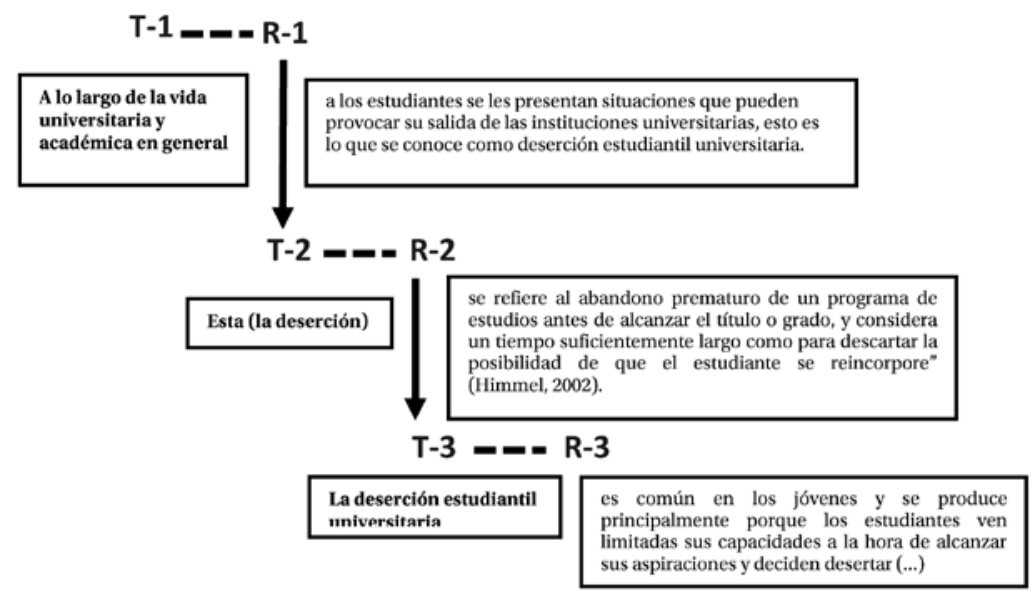

Figura 19. Progresión temática en el texto\#12 (Contexto 3)

Fuente: Elaboración propia.

Se puede apreciar que en el tema de la primera oración (T1), el estudiante proporciona una frase preposicional que indica una ubicación temporal para dar contexto al tema, mientras que en el rema (R1), identifica el fenómeno objeto de la explicación en el texto. Luego, en el tema de la oración 2 (T2), retoma el fenómeno introducido en el rema de la oración 1 y proporciona información para definirlo o conceptualizarlo, apoyándose en una cita. Más adelante, en la oración 3, retoma el mismo tema (T3) y agrega información en el rema (R3) para proporcionar información de contexto. Esta selección intencional de temas para enfatizar información y remas para proporcionar información nueva evidencia el conocimiento de diferentes patrones de progresión temática para lograr la coherencia. En el ejemplo se observa un patrón de progresión temática lineal entre las oraciones 1 y 2 , ya que parte del rema de la primera oración se convirtió en el tema de la siguiente. Mientras que entre las oraciones 2 y 3 se presenta un patrón

8 Según Halliday (1994), Tema es la información que se ubica al inicio de la oración; indica de qué se trata. Rema es la información nueva que se agrega al tema, es decir, lo que se dice acerca del Tema. 
de progresión temática constante, porque el tema de ambas oraciones es el mismo.

De igual manera, en los textos analizados se observa el empleo de recursos para dar cohesión al texto como otro mecanismo para lograr la coherencia, tales como referencia, reiteración, elipsis y marcadores textuales, entre otros (Halliday, 1994). En la Figura 21 se observa el uso de marcadores textuales para enlazar lógicamente partes del texto (por ejemplo: tales como...; De acuerdo con lo anterior...). También, se observa el uso de referencias para rastrear información ya mencionada en el texto (Otras de las variables...; en estos se puede...; para definirlos...). La reiteración se aplicó en el reemplazo de términos ya mencionados por sinónimos (variables-factores-agentes) o la repetición de términos para enfatizar, debido a su importancia en el desarrollo de la explicación del factor (estrés académico, institución, evaluación).

Otras de las variables influyentes en el estrés académico son los factores institucionales. Podemos definirlos como la relación entre los estudiantes y las instituciones educativas. Según Muñoz (2005), en estos se pueden resaltar tres agentes estresores principales: cómo los estudiantes afrontan las evaluaciones, la cantidad de trabajo académico que deben realizar y circunstancias agregadas de sus procesos universitarios, tales como la relación con los docentes, compañeros de clase, la dinámica del profesor en la clase, además de los factores de la institución como un plan de estudio defectuoso, el choque de horarios, entre otros (como se citó en Castrillón, Sarsosa \& Moreno, 2015). De acuerdo con lo anterior, los expertos resaltan la evaluación como uno de los factores más estresantes debido a la importancia que los estudiantes les dan. Es decir, los factores institucionales son elementos propios de la institución o suministrados por ésta, que generan cierto nivel de estrés en los estudiantes.

Figura 20. Ejemplo 10. Fragmento del texto \#12. Contexto 3

Además de los avances observados en el manejo de la estructura esquemática, características lingüísticas y discursivas de un texto de explicación factorial, los textos analizados también dan evidencias de apropiación de aspectos normativos y formales en cuanto a la gramática, ortografía y puntuación. Asimismo, se muestra el respeto por la propiedad intelectual, con la aplicación de las normas APA para la inclusión de citas en el ejemplo anterior. 


\section{Conclusiones}

Las universidades están llamadas a generar alternativas para proporcionar a los estudiantes de primer año las herramientas necesarias para insertarse con éxito en el contexto académico; para ello, la lectura y la escritura son fundamentales. El proyecto reportado en este artículo se inscribe dentro de estas alternativas. Su objetivo ha sido analizar la contribución de la pedagogía de géneros textuales, potenciada con técnicas de aprendizaje activo, en el engagement emocional y comportamental de los estudiantes, así como en el desarrollo de competencias de escritura académica.

La eficacia de la pedagogía de géneros textuales para el desarrollo de habilidades de lectura y escritura en los diferentes niveles de formación académica ha sido ampliamente comprobada. Sin embargo, su adaptación al contexto universitario ha dado lugar a variaciones en las fases del ciclo de enseñanza-aprendizaje para adaptarla a las necesidades específicas de los contextos (Dreyfus et al., 2015; Moss et al., 2016; Moyano, 2007, 2011). Las variaciones implementadas en el ciclo de escritura en el marco de este proyecto apuntaron a incentivar el engagement de los estudiantes durante las actividades de escritura y desarrollar habilidades de escritura académica.

Los resultados demuestran que las técnicas de aprendizaje activo incorporadas en las fases del ciclo de enseñanza de escritura propiciaron la participación activa de los estudiantes durante las interacciones, tanto con el docente como entre pares. Los organizadores gráficos y documentos interactivos utilizados durante la planificación, construcción y edición conjunta de los textos favorecieron la interacción e involucramiento de los estudiantes, especialmente en los trabajos de construcción y edición conjunta en pequeños grupos, mientras recibían explicación directa o retroalimentación de las docentes. Esto propició un clima de tranquilidad, seguridad y disfrute, lo que contribuyó a generar empatía por la asignatura y a moderar sus emociones ante el reto de escribir un texto académico. Se puede afirmar que la mayoría de los estudiantes adoptaron el rol como agentes activos en sus propios aprendizajes y asumieron los compromisos de la escritura académica con actitud positiva.

Asimismo, la propuesta influyó positivamente en la producción escrita de los estudiantes. Los textos analizados demuestran apropia- 
ción de la estructura y el lenguaje característico del género estudiado, así como la forma de configurar la información para alcanzar el propósito comunicativo. Igualmente, desarrollaron un registro formal, lo que demuestra conciencia del carácter abstracto y congruente del lenguaje de los textos académicos. De la misma manera, se apropiaron de recursos para dar cohesión y coherencia a sus textos.

A pesar de que las variables engagement y rendimiento académico fueron analizadas por separado en este estudio, los resultados sugieren una relación entre el engagement emocional y comportamental desarrollado por los estudiantes y el rendimiento alcanzado en la producción de sus textos. Se sugiere analizar esta relación asociada con la pedagogía de géneros textuales y sus implicaciones en futuros estudios.

\section{Referencias bibliográficas}

Álvarez, P. y Parra, A. (2015). Fortalecimiento de la expresión oral en un contexto de interacción comunicativa [Tesis de maestría no publicada]. Universidad Pedagógica y Tecnológica de Colombia.

Brame, C. (2016). Active learning. Vanderbilt University Center for Teaching. https://cft.vanderbilt.edu/active-learning/.

Benítez, T., Barletta, N., Chamorro, D., Mizuno, J., y Moss, G. (2018). Reading and writing across the curriculum. En A. Sellami y L. Fontaine, Perspectives from Systemic Functional Linguistics (pp. 86-108). Routledge.

Bernstein, B. (1990). La construcción social del discurso pedagógico. El Griot.

Bernstein, B. (1996). Pedagogy, symbolic, control and identity. Theory, research, critique. Rowman y Littlefield Publishers.

Barreyro, J. P., Injoque-Ricle, I., Álvarez-Drexler, A., Formoso, J., y Burin, D. (2016). Generación de inferencias explicativas en la comprensión de textos expositivos: el rol de la memoria de trabajo y el conocimiento previo específico. Suma Psicológica, 24(1), 17-24. https://doi.org/10.1016/j.sumpsi.2016.09.002

Bruner, J. S. (1987). Actual minds possible worlds. Harvard University Press. 
Carini, R., Kuh, G., y Klein, S. (2006). Student engagement and student learning: Testing the linkages. Research in Higher Education, 47(1), 1-32. https://doi.org/10.1007/s11162-005-8150-9

Carlino, P. (2005). Escribir, leer y aprender en la universidad. Una introducción a la alfabetización académica. Fondo de Cultura Económica.

Christie, F. y Martín, J. (Eds.). (1997). Genre and Institutions: Social processes in the workplace and school. Pinter.

Colectivo Comunicación (2002). Comprensión y competencias lectoras en estudiantes universitarios. Resultados de una investigación. Ediciones Uninorte.

Denzin, N. K. (1978). Sociological methods: A sourcebook. McGraw Hill.

Derewianka, B. y Jones, P. (2016). Teaching language in context. (2 ed.). Oxford University Press.

Dreyfus, S., Humphrey, S., Mahboob, A., y Martin, J. (2015). Genre pedagogy in higher education. Palgrave Macmillan.

Elliot, J. (1993). El cambio educativo desde la investigación-acción. Madrid: Morata.

Flórez, R. y Cuervo C. (2005). El regalo de la escritura, cómo aprender a escribir. Universidad Nacional de Colombia.

Fredricks, J., Blumenfeld, P., y Paris M. (2004). School engagement: Potential of the concept, state of the evidence. Review of Educational Research, 74(1), 59-100. https://doi. org/10.3102/00346543074001059

Gardner, M. K. (2012). Mixed-methods research. En J. R. Frankel, N. E. Wallen, y H. H. Hyun (Eds.), How to design and evaluate research in education (Eighth) (pp. 556-585). McGraw Hill.

González, H. 2000. El proyecto educativo de la Universidad Icesi y el aprendizaje activo (2a ed.). Universidad Icesi.

González, B., Salazar, A., y Peña, L. (2015). Formación inicial en lectura y escritura en la universidad. De la educación media al desempeño académico en la educación superior. Pontificia Universidad Javeriana.

Gunuc, S. (2014). The relationships between student engagement and their academic achievement. International Journal on New Trends in Education and Their Implications, 5(4), 199-214.

Halliday, M. A. K. (1994). An introduction to functional grammar. Routledge. 
Kemmis, S. and McTaggart, R. (1990). The action research planner. Victoria: Deakin University Press.

Kendeou, P. y van den Broek, P. (2005). The effects of readers' misconceptions on comprehension of scientific text. Journal of Educational Psychology, 97(2), 235-245. https://doi. org/10.1037/0022-0663.97.2.235

Latorre, A. (2005). La investigación acción. Conocer y cambiar la práctica educativa. Editorial Graó.

Lawson, M. y Lawson, H. (2013). New Conceptual frameworks for student engagement research, policy and practice. Review of Educational Research, 83(3), 432-479. https:// doi:10.3102/0034654313480891

Leech, N. L. y Onwuegbuzie, A.J. (2009). A typology of mixed methods research designs. Qual Quant, 43(2), 265-275. https://doi. org/10.1007/s11135-007-9105-3

Linnenbrink, E. y Pintrich, P. (2003a). Motivation as an enabler for academic success. School Psychology Review, 31(3), 313-327. https://doi.org/10.1080/02796015.2002.12086158

Linnenbrink, E. y Pintrich, P. (2003b). The role of self-efficacy beliefs in student engagement and learning in the classroom. Reading y Writing Quarterly: Overcoming Learning Difficulties, 19(2), 119-137. https://doi.org/10.1080/10573560308223.

Martin, J. R. (1992). English text: System and structure. John Benjamins.

Martin, J. R. y Rose, D. (2005). Designing literacy pedagogy: Scaffolding democracy in the classroom. En R. Hasan, C. Matthiessen, y J. Webster (Eds.), Continuing discourse on language: $A$ functional perspective (pp. 252-280). Equinox.

Martin, J. R. y Rose, D. (2008). Genre relations: Mapping culture. Equinox.

Moss, G., Benítez, T., y Mizuno, J. (Eds.). (2016). Textos que se leen y escriben en la universidad: una mirada desde los géneros discursivos. Universidad del Norte.

Moyano, E. I. (2007). Enseñanza de habilidades discursivas en español en contextos pre-universitarios: una aproximación desde la LSF. Revista Signos, 40(65), 573-608. https://doi.org/10.4067/ s0718-09342007000300009

Moyano, E. I. (16-19 de agosto de 2011). Deconstrucción y edición conjuntas en la enseñanza de la escritura: la reflexión sobre gé- 
nero y discurso en la formación académica y profesional. VI Simposio Internacional de Estudios de Géneros Textuais (VI SIGET). Universidade Federal do Rio Grande do Norte, Brasil.

Moyano, E. I. (2013). Una didáctica de las ciencias basada en los géneros textuales: acceso a las disciplinas a través de la apropiación de su discurso. En E. I. Moyano (Coord.), Aprender ciencias y humanidades: una cuestión de lectura y escritura. Aportes para la construcción de un programa de inclusión social a través de la educación lingüística (pp. 109-155). UNGS.

Moyano, E. I. (2017). Diseño e implementación de programas de lectura y escritura en el nivel universitario: principios y estrategias. Lenguas Modernas, 50, 47-72.

Olave-Arias, G., Cisneros-Estupiñán, M., y Rojas-García, I. (2013). Deserción universitaria y alfabetización académica. Educación y Educadores, 16(3), 455-471. https://doi.org/10.5294/ edu.2013.16.3.4

Pérez, M. y Rincón, G. (2013). ¿Para qué se lee y se escribe en la universidad colombiana? Un aporte a la consolidación de la cultura académica del país. Editorial Pontificia Universidad Javeriana.

Reeve, J. (2003). Motivación y emoción. Mcgraw-Hill.

Rose, D. (2004). Sequencing and pacing of the hidden curriculum: How indigenous learners are left out of the chain. En J. A. Muller y B. Davies Morais (Eds.), Reading Bernstein, researching Bernstein (pp. 91-107). Routledge.

Rose, D. y Acevedo, C. (2006). Closing the gap and accelerating learning in the middle years of schooling. Australian Journal of Language and Literacy, 14(2), 32-45.

Rose, D. y Martin, J. R. (2012). Learning to write, reading to learn. Genre, knowledge and pedagogy in the Sydney School. Equinox.

Rothery, J. y Stenglin, M. (1994). Spine-chilling stories: A unit of work for junior secondary English. Metropolitan East Disadvantaged Schools Program.

Salonava, M., Martínez, I., Bresó, E., Llorens, S., y Gumbau, R. (2005). Bienestar psicológico en estudiantes universitarios: facilitadores y obstaculizadores del desempeño académico. CSIC. Anales de Psicología, 1(21), 170-180. 
Sivan, A., Wong, R., Chi-Ching, W., y Kember, D. (2010). An implementation of active learning and its effect on the quality of student learning. Innovation in Education and Training International, 37(4), 381-389. https://doi. org/10.1080/135580000750052991

Trowler, P. y Trowler, V. (2010). Frameworks for action: enhancing student engagement at the institutional level. The Higher Education Academy.

Umbach, P. y Wawrzynski, M. (2005). Faculty do Matter: The Role of College Faculty in Student Learning and Engagement. Research in Higher Education, 46(2), 153. https://doi.org/10.1007/ s11162-004-1598-1.

Vygotsky, L. (1962). Thought and language. Translated by Eugenia Hanfmann (1986). MIT Press.

Whitehead, J. (1999). How do I improve my practice? Creating a new discipline of educational enquiry [Doctoral dissertation]. http://www.actionresearch.net/living/jackwhitehead2.sh.

Zwaan, R. y Rapp, D. (2006). Discourse comprehension. In M. A. Gernsbacher y M. J. Traxler (Eds.), Handbook of psycholinguistics (pp. 725-764). Elsevier. 


\section{Anexo 1: Organizador texto de explicación factorial}

\begin{tabular}{ll}
\hline \multicolumn{1}{c}{ ETAPA: EXPLICACIÓN DE FACTORES } \\
\multicolumn{1}{c}{ FASE: FACTORES INSTITUCIONALES: RELACIÓN DOCENTE-ESTUDIANTE }
\end{tabular}




\begin{tabular}{|c|c|}
\hline $\begin{array}{l}\text { Explica diferentes aspectos } \\
\text { relacionados con el factor } \\
\text { que muestran cómo este } \\
\text { influye en el rendimiento } \\
\text { académico: } \\
\text { - en qué consiste }\end{array}$ & $\begin{array}{l}\text { La relación docente-estudiante comprende el vínculo } \\
\text { que existe entre estos dos sujetos, en donde el docente } \\
\text { lograr marcar y tener gran influencia en el estudian- } \\
\text { te. (Mas, 2012). Es decir, el docente es una autoridad } \\
\text { y modelo con el que el estudiante está continuamente } \\
\text { conviviendo, por lo que este último se verá afectado y } \\
\text { tendrá un impacto en distintos aspectos. }\end{array}$ \\
\hline $\begin{array}{l}\text { - cómo se reconoce } \\
\text { - cómo causa o influye }\end{array}$ & $\begin{array}{l}\text { La relación docente estudiantes depende de diferentes } \\
\text { aspectos tales como: el número de horas compartidas, } \\
\text { la forma de enseñanza del docente y el ambiente gene- } \\
\text { rado en el aula de clase (Mas, 2012). }\end{array}$ \\
\hline $\begin{array}{l}\text { académico. } \\
\text { - Cita investigaciones que } \\
\text { evidencian cómo el factor } \\
\text { influye en el rendimiento } \\
\text { académico. } \\
\text { En la explicación cita } \\
\text { diferentes autores que han }\end{array}$ & $\begin{array}{l}\text { Dentro de los salones de clase es donde los docentes } \\
\text { dan a conocer sus recursos personales y didácticos } \\
\text { con el fin de hacer su trabajo de la mejor manera, para } \\
\text { así poder establecer relación con los estudiantes, pero } \\
\text { como todas relaciones humanas, esta tiene caracterís- } \\
\text { ticas implícitas y explícitas que la hacen diferente de } \\
\text { cualquier otra relación interpersonal (Cámere, 2009). }\end{array}$ \\
\hline $\begin{array}{l}\text { investigado sobre el tema, } \\
\text { e incluye su propia voz } \\
\text { interpretando o explicando } \\
\text { al menos una de las citas. } \\
\text { (3-4 oraciones) }\end{array}$ & $\begin{array}{l}\text { La relación que se genera entre el alumno y el docen- } \\
\text { te es de gran importancia, para garantizar el éxito del } \\
\text { proceso de enseñanza-aprendizaje y poder dar frente } \\
\text { a la superación de ciertos obstáculos como son los di- } \\
\text { ferentes niveles y ritmos de atención, concentración, } \\
\text { aprendizaje y desarrollo de los estudiantes (Vargas, } \\
2019 \text { ). }\end{array}$ \\
\hline $\begin{array}{l}\text { Concluye con una } \\
\text { afirmación/declaración o } \\
\text { comentario general para } \\
\text { dar cierre al párrafo. } \\
\text { (1 oración) }\end{array}$ & $\begin{array}{l}\text { Aquellos alumnos que logran desarrollar una relación } \\
\text { con sus profesores, aprenden con mayor facilidad den- } \\
\text { tro del contexto académico, pues son estudiantes se- } \\
\text { guros de sí mismos, capaces de superar diferentes obs- } \\
\text { táculos y que se encuentran en constante aprendizaje } \\
\text { en compañía de sus maestros. }\end{array}$ \\
\hline
\end{tabular}

REFERENCIAS

Vargas, M. R. (21 de 01 de 2019). La Nación en Noticia Independiente. Obtenido de https://www. lanacion.com.co/relacion-docente-alumnoelemento-fundamental-para-el-proceso-deaprendizaje/

Cámere, E. (01 de 08 de 2009). Entre Educadores Educación Familia y Valores. Obtenido de https:// entreeducadores.com/2009/08/01/la-relacion-profesor-alumno-en-el-aula/

Kopyto, I. (2016). Universidad de Palermo. Obtenido de https://fido.palermo.edu/servicios_dyc/ publicacionesdc/vista/detalle_articulo. php?id_libro $=684 \& i d \_a r t i c u l o=14260$

Educación 3.0. (26 de 11 de 2019). Educación 3.0 Líder Informativo en Innovación Educativa. Obtenido de https://www.educaciontrespuntocero. com/noticias/por-que-vinculo-estudiantesdocentes-es-clave-aprender/

Fuente. Elaboración propia. 


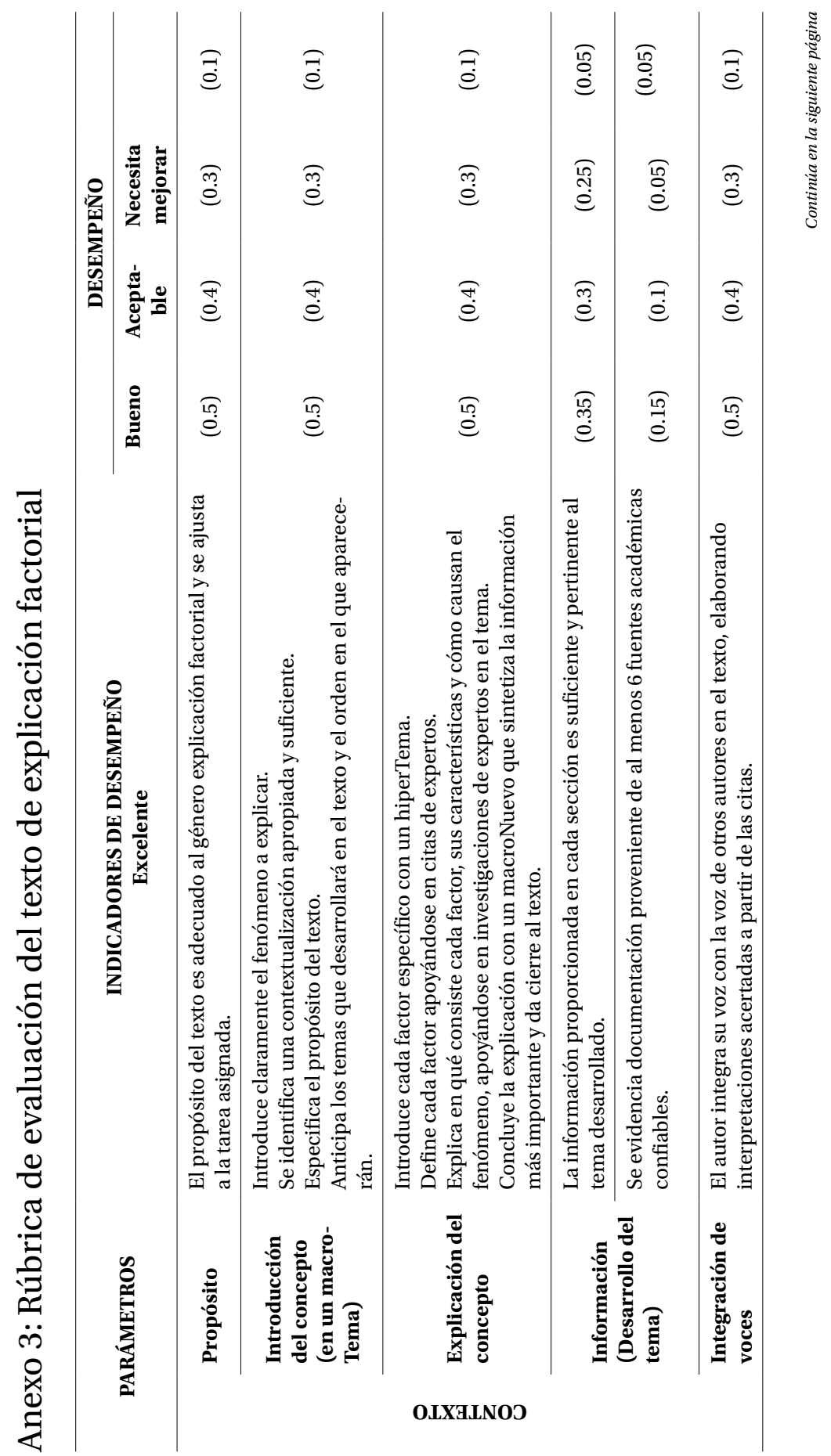




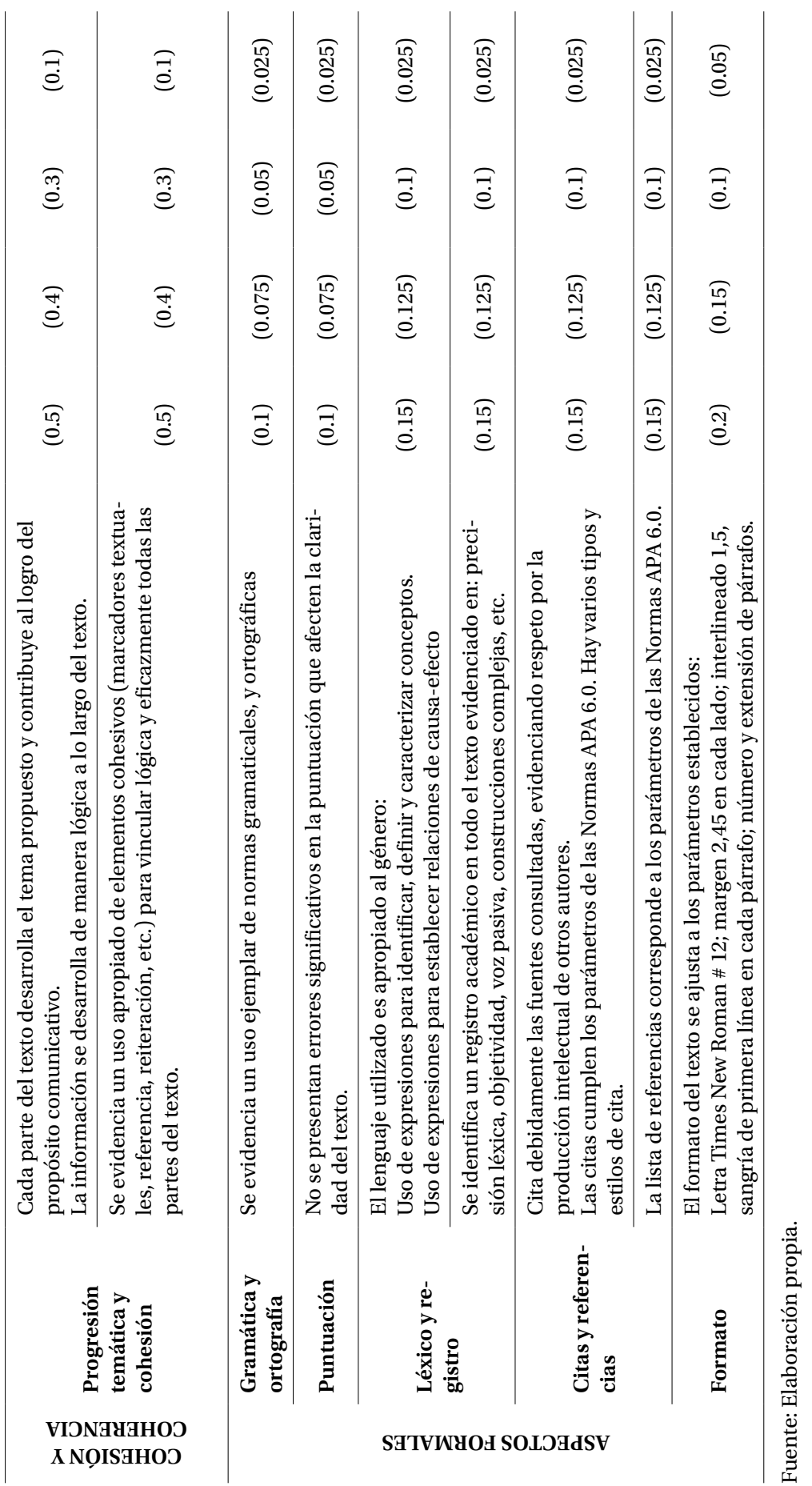




\section{Anexo 2. Texto de explicación factorial académico de estudiante}

\section{Deserción estudiantil ea el contexto universitario}

La deserciōn estudiantil universitaria se define como "el fenómeno en el cual un estudiante no logra cumplir sus metas edacativas, y presenta inactividad académica por un aho o más" (Chain, 2001, p. 28). Este vendria siendo uno de los principales problemas que enfrenta la educación superior debido a las repercusiones institucionales, sociales y personales que abarcan este tema. Por esta razón se analizarán los distintos factores causantes de la deserciön de estudiantes universitarios tales como, factores personales, es decir las metas y el ajuste social e institucional, y los factores acadímicos que abarcan la deficiente orientación vocacional, la falta de habilidades y la formación previa a la universidad.

Uno de los principales factores cansantes de la deserción estudiantil es el académico, donde se evidencia la deficiente o nula orientación socacional, la influencia de padres o amigos, las expectativas de la carrera no cubiertas, la falta de habilidades y la formación previa a la universidad. Investigaciones demuestran que un alto nùmero de estudiantes que abandonaron sus estudios, no tenian claro sus planes académicos al momento de la deserción. Esto da a conocer que la mayoria de los estudiantes que ingresan a la universidad tienen poca información de la carrera que cursan. No tienen conocimiento de las materias de su curriculo, su alcance laboral y no cuentan con la motivación y herramientas necesarias para superar la carrera que han elegido. El modelo sociológico de Spady (1970, p. 14) explica que 7a decisión de desertur obedece a la dificultad de integrarse con el entorno de la educación superior"; aqui adquiere enorme importancia el ambiente familiar porque deteriora el potencial académico. Es decir, la educación ofrecida antes de la universidad tanto en una institución como en la familia serian los principales influyentes en el éxito o fracaso futuro del estudiante.

Otro factor principal, vendria siendo el personal que tiene que ver con el ajuste social e institucional y las metas personales del estudiante. A veces los estudiantes deciden conchuir sus estudios ya que no tienea clara una meta o simplemente no quieren seguir estudiando. "Es importante reconocer que ciertas per sonas deciden a veces abandonar las instituciones de educación superior, sin alcanzar la graduación, sencillamente porque so tienen la meta o la intención de permanecer en ella hasta lograr esa graduación" (Tinto, 1994, p. 459). Teniendo en cuenta esta ùltima frase cuando el alumno carece de sus objetivos, metas y plan de vida a futuro, es más costoso que siga sus estudios universitarios y los conchrya.

En cuanto a los principales problemas que enfrenta la educación soperior, la deserción eitudiantil es de suma importancia. De aqui viene la necesidad de identificar los factores causantes y atacar el problema con el fin de que los estudiantes busquen formas en las que puedan adoptarse mejor al ambiente universitario. Por esto, es necesario incentivar a los docentes a no estigmatizar el cambio de carrera y enfocar la educación a un proyecto a futuro, y a los padres a ayudar a sus bijos en sa orientación vocacional, en vez de exigitles una deteminada carrera.

\section{Referencias}

Canales, A, \& De los Rios, D. (2018). Factores explicativos de la deserción universitaria Calidad en la Educación, (26).

Fiegeben, L. (2005). Repitencia y deserción universitaria en América Latina. Presentado en el Sewninario de Edhación Superior de América Latina y el Caribe. Capitulo, II.

Mori Sánchez, M. D. P. (2014). Deserción universitaria en estudiantes de una universidad privada de Iquitos. Resista Digital de Imvestigación en Docencia Univerzifaria (RIDU).

Ramirez, G. \& Corvo, M. (2007). Causas de deserción de alumnos de primetos semestres de una unkersidad privada Revista Mericana de Ortentación Edhcatrva, 5(12), 34-39.

Tinto, V. (1975). Descrción en educación superior. Sintesis teóticas de investigaciones recientes; revision de investigaciones educacionales, 45(1), 89-12

Fuente: Elaboración propia. 